\title{
Jetzt bewerben: Werner-Porstmann-Preis zur Förderung der interventionellen Radiologie
}

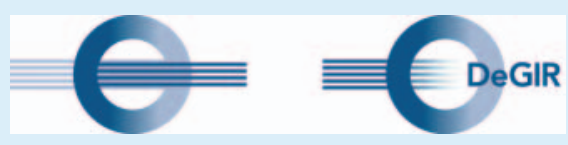

In Gedenken an Werner Porstmann (19211982), einen Pionier der kardiovaskulären Interventionen, schreiben die Deutsche Röntgengesellschaft und die Deutsche Gesellschaft für Interventionelle Radiologie und minimalinvasive Therapie (DeGIR) jährlich den Werner-Porstmann-Preis zur Förderung der interventionellen Radiologie aus.

\section{Auswahlkriterien}

Der Preis wird verliehen für die beste wissenschaftliche Arbeit auf dem Gebiet der interventionellen Radiologie, die in den 2 der Verleihung vorausgehenden Jahren veröffentlicht beziehungsweise zur Veröffentlichung angenommen wurde. Der Preis wird von der Terumo Deutschland $\mathrm{GmbH}$ unterstützt und ist mit 1500 Euro dotiert. Der einreichende Autor/die einreichende Autorin muss Mitglied der DRG und der DeGIR sein. Den Hauptteil der zugrunde liegenden Studie sollte der Bewerber/die Bewerberin hauptsächlich selbstständig durchgeführt haben. Es darf nur eine Publikation eingereicht werden.

\section{Bewerbungsunterlagen und Fristen}

Die Bewerbungsunterlagen enthalten die wissenschaftliche Arbeit, einen kurzen
Lebenslauf sowie ein Schriftenverzeichnis der letzten 2 Jahre, an denen der einreichende Autor/die einreichende Autorin als Erst- oder Letztautor/in beteiligt war. Bei Veröffentlichungen mit mehreren Autor/ innen ist der Bewerbung eine Bestätigung der vorgesetzten Person beizufügen, die ausweist, dass der einreichende Autor/die einreichende Autorin den entscheidenden Anteil an der Veröffentlichung trägt.

Bitte reichen Sie die Bewerbungsunterlagen per E-Mail in elektronischer Form (PDF-Dokument) bis zum 30. Juni 2021 an Frau Ermisch in der DRGGeschäftsstelle ein: ermisch@drg.de. 